\title{
Parallel Adaptive Mesh Refinement Scheme for Three-Dimensional Turbulent Non-Premixed Combustion
}

\author{
X. Gao* and C. P. T. Groth ${ }^{\dagger}$ \\ University of Toronto Institute for Aerospace Studies \\ 4925 Dufferin Street, Toronto, Ontario, M3H 5T6, Canada
}

\begin{abstract}
A parallel adaptive mesh refinement (AMR) algorithm is described for predicting turbulent non-premixed gaseous combusting flows in three space dimensions. The Favreaveraged Navier-Stokes equations governing a reactive mixture of thermally perfect gases, the two transport equations of the $k-\omega$ turbulence model, and the time-averaged species transport equations, are all solved using a fully coupled finite-volume formulation on bodyfitted multi-block hexahedral mesh. The numerical algorithm adopts a cell-centred upwind finite-volume discretization procedure and uses limited solution reconstruction, approximate Riemann solver based flux functions to determine the inviscid (hyperbolic) flux at cell interfaces. The viscous (elliptic) components of the cell face flux are evaluated by employing a hybrid average gradient-diamond path approach. For the treatment of near-wall turbulence, both low-Reynolds-number and wall-function formulations of the $k$ - $\omega$ model are used, with a procedure for automatically switching from one to the other, depending on mesh resolution. A flexible block-based hierarchical octree data structure is used to maintain the connectivity of the solution blocks in the multi-block mesh and facilitate automatic solution-directed mesh adaptation according to physics-based refinement criteria. This AMR approach allows for anisotropic mesh refinement and the block-based data structure readily permits efficient and scalable implementations of the algorithm on multi-processor architectures. Numerical results for turbulent non-premixed methane-air diffusion flames are described to demonstrate the validity and potential of the parallel AMR approach for predicting complex combusting flows.
\end{abstract}

\section{Introduction}

With recent advances in computational fluid dynamics (CFD) and numerical methods for combusting flows, as well as advances in high-performance-computing hardware, numerical modelling has become an important powerful and effective tool for the design of advanced combustion systems. The importance of numerical modelling has increased with the increasingly stringent emission legislation imposed by governments worldwide. The latter has made the combustor and engine design process much more challenging. As virtually all practical combustion systems involve turbulent combustion and pollutant and particulate emissions are controlled by the details of the turbulent fuel-air mixing and combustion processes, a detailed understanding of the strong nonlinear interaction between the turbulent flow structure, chemical kinetics, and thermodynamic properties of the reactants and products is required for obtaining improved low-emission combutor designs.

Three primary tools for performing simulations of turbulent combusting flows have emerged: (i) direct numerical simulation (DNS); (ii) large-eddy simulation (LES); (iii) and Reynolds- or Favre-averaged NavierStokes (RANS) simulation techniques, each possessing various advantages and disadvantages. ${ }^{1,2}$ In DNS, all of the turbulent and chemical length and time scales are fully resolved. For this reason, DNS is a powerful tool for studying turbulent flame structure and turbulence/chemistry interactions in detail. It is mainly reserved for understanding the basic processes of combustion phenomena, such as extinction and re-ignition, flow and

*PhD Candidate, Email: gao@utias.utoronto.ca, Student Member AIAA

$\dagger$ Associate Professor, Email: groth@utias.utoronto.ca, Senior Member AIAA 
flame unsteadiness, and differential diffusion of chemical species (some recent examples of the application of DNS techniques to problems in combustion are described by Vervisch ${ }^{3}$ ). However, despite the successes to date, DNS is generally restricted to generic simplified and/or more academic combustor configurations due to the very high computational costs of fully resolving all solution scales. It will not be used to simulate turbulent combustion phenomena in practical combustor configurations with complex geometry any time in the near future. LES is an alternative to DNS in which the large energy containing structures or eddies are computed directly and the small and generally more universal dissipative turbulent scales are modelled, thereby offering potential computational savings. ${ }^{4}$ Over the last decade, the approach has evolved to become a truly predictive tool for non-reacting flows ${ }^{4-8}$ and has been shown in many applications to provide more accurate predictions of the flow fields than the more conventional RANS-based methods for reacting flows. ${ }^{3}$ Nevertheless, universal and accurate sub-filter scale models for non-premixed and premixed reacting flows are not currently available and the accurate and reliable numerical solution of the filtered Navier-Stokes equations remains a significant computational challenge for many practical problems in turbulent combustion.

As LES is still at an early stage of development for combusting flows and due to the still relatively high cost of performing such simulations, RANS-based methods are the predominant approach in engineering CFD applications for combusting flows involving complex flow geometries. ${ }^{9}$ This situation is not expected to change in the near future. Nevertheless, in spite of simplifications offered by time-averaging approaches, the system of equations governing turbulent combusting flows can be both large and stiff and its solution can still place severe demands on available computational resources. In particular, approaches are required to reduce the computational costs of simulating combusting flows using RANS-based methods, thereby permitting their application on a more routine basis.

Many approaches have been taken to reduce the computational costs of simulating combusting flows. One successful approach is to make use of solution-directed mesh adaptation, such as the adaptive mesh refinement (AMR) algorithms. Originally proposed by Berger and Oliger for computing time-dependent solutions to hyperbolic partial differential equations in multiple space dimensions, ${ }^{10}$ AMR approaches have been developed for a wide variety of engineering problems. ${ }^{11-29}$ AMR is a powerful tool for computing solutions to partial differential equations whose solutions have disparate spatial scales. Computational grids are automatically adapted to the solution of the governing equations and this is very effective in treating problems with multiple scales, providing the required spatial resolution while minimizing memory and storage requirements. Another approach for coping with the computational cost of reacting flow prediction is to apply a domain decomposition procedure and solve the problem in a parallel fashion using multiple processors. Large massively parallel distributed-memory computers can provide many fold increases in processing power and memory resources beyond those of conventional single-processor computers and would therefore provide an obvious avenue for greatly reducing the time required to obtain numerical solutions of combusting flows (see Douglas et al. ${ }^{30}$ Reynolds and Fatica, ${ }^{31}$ and Medic et al. ${ }^{32}$ for examples of parallel algorithm development for numerical combustion modelling). Finally, there is also the possibility of reducing computational costs by combining a parallel implementation with an AMR strategy, producing a parallel AMR method that both reduces the overall problem size and the time to calculate a solution. Recent progress in the development and application of parallel AMR algorithms for low-Mach-number reacting flows and premixed turbulent combustion is described by Day and Bell ${ }^{33-35}$ and more recently, Northrup and Groth ${ }^{36}$ and Gao and Groth ${ }^{37,38}$ have also proposed a parallel block-base AMR method using body-fitted multi-block mesh for application to both laminar and turbulent non-premixed combusting flows.

This paper describes the direct extension of the parallel algorithm proposed in the previous work of Gao and Groth ${ }^{37,38}$ for two-dimensional planar and axisymmetric turbulent non-premixed combusting flows to the three-dimensional case using body-fitted multi-block hexahedral mesh. The paper is structured as follows. In Section II, the system of governing equations for a compressible thermally perfect reactive mixture of gases is presented. In Section III, the main elements of the numerical algorithm are described. Numerical validation of the algorithm is performed in Section IV. Components of the proposed algorithm are are evaluated separately for several canonical flow problems. In Section V, the solutions of the three-dimensional code for a bluff-body burner are compared to experimental results. Finally, the parallel performance of the proposed algorithm is considered in Section VI and conclusions are drawn in Section VII. 


\section{Mathematical Model of Turbulent Combusting Flows}

\section{II.A. Favre-Averaged Navier-Stokes Equations}

A mathematical model based on the Favre-averaged Navier-Stokes equations for a compressible thermally perfect reactive mixture of gases has been formulated and is used herein to describe turbulent non-premixed combustion processes. In this formulation, the continuity, momentum, and energy equations for the reactive mixture of $N$ species are

$$
\begin{gathered}
\frac{\partial \rho}{\partial t}+\nabla \cdot(\rho \overrightarrow{\mathbf{u}})=0 \\
\frac{\partial}{\partial t}(\rho \overrightarrow{\mathbf{u}})+\nabla \cdot(\rho \overrightarrow{\mathbf{u}} \overrightarrow{\mathbf{u}}+p \overrightarrow{\overrightarrow{\mathbf{I}}})=\nabla \cdot(\overrightarrow{\boldsymbol{\tau}}+\overrightarrow{\vec{\lambda}})+\overrightarrow{\mathbf{f}} \\
\frac{\partial}{\partial t}(\rho e)+\nabla \cdot\left[\rho \overrightarrow{\mathbf{u}}\left(e+\frac{p}{\rho}\right)\right]=\nabla \cdot[(\overrightarrow{\vec{\tau}}+\overrightarrow{\vec{\lambda}}) \cdot \overrightarrow{\mathbf{u}}]+\nabla \cdot\left(D_{k} \boldsymbol{\nabla} k\right)-\nabla \cdot\left(\overrightarrow{\mathbf{q}}+\overrightarrow{\mathbf{q}}_{t}\right)+\overrightarrow{\mathbf{u}} \cdot \overrightarrow{\mathbf{f}},
\end{gathered}
$$

where $\rho$ is the time-averaged mixture density, $\overrightarrow{\mathbf{u}}$ is the Favre-averaged mean velocity of the mixture, $p$ is the time-averaged mixture pressure, $e=|\overrightarrow{\mathbf{u}}|^{2} / 2+\sum_{n=1}^{N} c_{n} h_{n}-p / \rho+k$ is the Favre-averaged total specific mixture energy, $\overrightarrow{\mathbf{f}}$ is a body force per unit volume acting on the gaseous mixture, $k$ is the specific turbulent kinetic energy, $D_{k}$ is the coefficient for the diffusion of the turbulent energy, $\overrightarrow{\boldsymbol{\tau}}$ and $\overrightarrow{\vec{\lambda}}$ are the molecular and turbulent Reynolds stress tensors or diads, and $\overrightarrow{\mathbf{q}}$ and $\overrightarrow{\mathbf{q}}_{t}$ are the molecular and turbulent heat flux vectors, respectively. The mixture pressure is given by the ideal gas law

$$
p=\sum_{n=1}^{N} \rho c_{n} R_{n} T,
$$

where $R_{n}$ is the species gas constant and $T$ is the mixture temperature. Fourier's law is used to represent the thermal diffusion caused by the random thermal motion and turbulence. In addition, $h_{n}$ is the absolute (chemical and sensible) internal enthalpy for species $n$. The transport equation describing the time evolution of the species mass fraction, $c_{n}$, is given by

$$
\frac{\partial}{\partial t}\left(\rho c_{n}\right)+\nabla \cdot\left(\rho c_{n} \overrightarrow{\mathbf{u}}\right)=-\nabla \cdot\left(\overrightarrow{\mathcal{J}}_{n}+\overrightarrow{\mathcal{J}}_{t n}\right)+\rho \dot{w}_{n},
$$

where $\dot{w}_{n}$ is the time-averaged or mean rate of the change of the species mass fraction produced by the chemical reactions and $\overrightarrow{\mathcal{J}}_{n}$ and $\overrightarrow{\mathcal{J}}_{t n}$ are the molecular and turbulent diffusive fluxes for species $n$, respectively. The latter are specified using Fick's law.

\section{II.B. Turbulence Model}

The modified two-equation $k-\omega$ model of Wilcox ${ }^{39}$ is used here to model the unresolved turbulent flow quantities. In this approach, the Boussinesq approximation is used to relate the Reynolds stress tensor, $\overrightarrow{\vec{\lambda}}$, to the mean flow strain-rate tensor using a turbulent eddy viscosity, $\mu_{t}$,

$$
\lambda_{i j}=\mu_{t}\left[\frac{\partial u_{i}}{\partial x_{j}}+\frac{\partial u_{j}}{\partial x_{i}}-\frac{2}{3} \delta_{i j} \frac{\partial u_{k}}{\partial x_{k}}\right]-\frac{2}{3} \delta_{i j} \rho k
$$

with $\mu_{t}=\rho k / \omega$. Transport equations are solved for turbulent kinetic energy, $k$, and the specific dissipation rate, $\omega$, given by

$$
\begin{gathered}
\frac{\partial}{\partial t}(\rho k)+\nabla \cdot(\rho k \overrightarrow{\mathbf{u}})=\overrightarrow{\vec{\lambda}}: \nabla \overrightarrow{\mathbf{u}}+\nabla \cdot\left[\left(\mu+\mu_{t} \sigma^{*}\right) \nabla k\right]-\beta^{*} \rho k \omega, \\
\frac{\partial}{\partial t}(\rho \omega)+\nabla \cdot(\rho \omega \overrightarrow{\mathbf{u}})=\alpha \frac{\omega}{k} \overrightarrow{\vec{\lambda}}: \nabla \overrightarrow{\mathbf{u}}+\nabla \cdot\left[\left(\mu+\mu_{t} \sigma\right) \nabla \omega\right]-\beta \rho \omega^{2}
\end{gathered}
$$

where $\mu$ is the molecular viscosity of the mixture and $\sigma^{*}, \beta^{*}, \alpha, \sigma$, and $\beta$ are closure coefficients for the two-equation model. The latter are given by

$$
\alpha=\frac{13}{25}, \quad \beta=\beta_{\circ} f_{\beta}, \quad \beta^{*}=\beta_{\circ}^{*} f_{\beta^{*}}, \quad \sigma=\sigma^{*}=\frac{1}{2},
$$


with

$$
\begin{gathered}
\beta_{\circ}=\frac{9}{125}, \quad \beta_{\circ}^{*}=\frac{9}{100}, \\
f_{\beta}=\frac{1+70 \chi_{\omega}}{1+80 \chi_{\omega}}, \quad f_{\beta^{*}}=\left\{\begin{array}{ll}
1 & \chi_{k} \leq 0, \\
\frac{1+680 \chi_{k}^{2}}{1+400 \chi_{k}^{2}} & \chi_{k}>0,
\end{array},\right.
\end{gathered}
$$

and

$$
\chi_{\omega}=\left|\frac{\Omega_{i j} \Omega_{j k} S_{k i}}{\left(\beta_{\circ}^{*} \omega\right)^{3}}\right|, \quad \chi_{k}=\frac{1}{\omega^{3}} \frac{\partial k}{\partial x_{j}} \frac{\partial \omega}{\partial x_{j}} .
$$

The tensors $\Omega_{i j}$ and $S_{k i}$ are the vorticity and strain rate tensors, respectively.

Both low-Reynolds-number and wall-function formulations of the $k-\omega$ model are used for the treatment of near-wall turbulent flows, with a procedure for automatically switching from one to the other, depending on mesh resolution. In the case of the low-Reynolds-number formulation, it can be shown that

$$
\lim _{y \rightarrow 0} \omega=\frac{6 \nu}{\beta y^{2}},
$$

where $y$ is the distance normal from the wall. ${ }^{39}$ Rather than attempting to solve the $\omega$-equation directly, the preceding expression is used to specify $\omega$ for all values of $y^{+} \leq 2.5$, where $y^{+}=u_{\tau} y / \nu, u_{\tau}^{2}=\tau_{w} / \rho$, and $\tau_{w}$ is the wall shear stress. Provided there are 3-5 computational cells inside $y^{+}=2.5$, this procedure reduces numerical stiffness, guarantees numerical accuracy, and permits the $k-\omega$ model to be solved directly in the near-wall region without resorting to wall functions. In the case of the wall-function formulation, the expressions

$$
\begin{gathered}
\frac{u}{u_{\tau}}=\frac{1}{\kappa} \ln \left(y^{+}\right)+C, \\
k=\frac{u_{\tau}^{2}}{\sqrt{\beta^{*}}}, \\
\omega=\frac{u_{\tau}}{\sqrt{\beta^{*}} \kappa y},
\end{gathered}
$$

are used to fully specify $k$ and $\omega$ for $y^{+} \leq 30-250$, where $\kappa$ is the von Kármán constant. A procedure has been developed to automatically switch between these two approaches, depending on the near-wall mesh resolution. This automatic near-wall treatment readily accommodates situations during adaptive mesh refinement where the mesh resolution may not be sufficient for directly calculating near-wall turbulence using the low-Reynolds-number formulation.

\section{II.C. Thermodynamic and Transport Properties}

Thermodynamic relationships and transport coefficients are required to close the system of equations given above. Thermodynamic and molecular transport properties of each gaseous species are prescribed using the empirical database compiled by Gordon and McBride, ${ }^{40,41}$ which provides curve fits for the species enthalpy, $h_{n}$; specific heat, $c_{p_{n}}$; entropy; viscosity, $\mu_{n}$; and thermal conductivity, $\kappa_{n}$, as functions of temperature, $T$. For example, the enthalpy and viscosity for a particular species is given by

$$
\begin{gathered}
h_{n}=R_{n} T\left[-a_{1, n} T^{-2}+a_{2, n} T^{-1} \ln T+a_{3, n}+\frac{a_{4, n}}{2} T+\frac{a_{5, n}}{3} T^{2}+\frac{a_{6, n}}{4} T^{3}+\frac{a_{7, n}}{5} T^{4}+b_{1} T^{-1}\right]+\Delta h_{f_{n}}^{o}, \\
\ln \mu_{n}=A_{n} \ln T+\frac{B_{n}}{T}+\frac{C_{n}}{T^{2}}+D_{n},
\end{gathered}
$$

where $a_{k, n}, A_{n}, B_{n}, C_{n}$, and $D_{n}$ are the coefficients for the curve fits. The Gordon-McBride data set contains curve fits for over 2000 substances, including 50 reference elements.

The molecular viscosity, $\mu$, and thermal conductivity, $\kappa$, of the reactive mixture are determined using the mixture rules of Wilke ${ }^{42}$ and Mason and Saxena, ${ }^{43}$ respectively. Turbulent contributions to thermal conductivity and species diffusivity are modelled by making an analogy between momentum and heat transfer and introducing the turbulent Prandtl and Schmidt numbers, $\mathrm{Pr}_{t}$ and $\mathrm{Sc}_{t}$, both of which are taken to be constant $\left(\mathrm{Pr}_{t}=0.9\right.$ and $\left.\mathrm{Sc}_{t}=1\right)$, and assuming $\kappa_{t}=\mu_{t} c_{p} / \mathrm{Pr}_{t}$ and $D_{t n}=\mu_{t} / \rho \mathrm{Sc}_{t}$. 


\section{II.D. Reduced Chemical Kinetics and Eddy Dissipation Model}

The primary goal of this research is to establish a computational framework for predicting complex reacting flows in practical combustor geometries. For these purposes, the use of simplified chemical mechanisms for gaseous fuels and turbulence-chemistry interaction model allowed for the validation of the proposed solution algorithm without the added complexities and computational overhead of dealing with more complex mechanisms and turbulence-chemistry interaction models. Future research will involve the incorporation of more sophisticated combustion modelling, as well as modelling for liquid fuels and radiation transport.

For the gaseous methane-air combustion considered in the present work, the following reduced, one-step, five-species, chemical kinetic scheme of Westbrook and Dryer ${ }^{44}$ is used:

$$
\mathrm{CH}_{4}+2 \mathrm{O}_{2} \rightarrow \mathrm{CO}_{2}+2 \mathrm{H}_{2} \mathrm{O} .
$$

The five species are methane $\left(\mathrm{CH}_{4}\right)$, oxygen $\left(\mathrm{O}_{2}\right)$, carbon dioxide $\left(\mathrm{CO}_{2}\right)$, water $\left(\mathrm{H}_{2} \mathrm{O}\right)$, and nitrogen $\left(\mathrm{N}_{2}\right)$. Nitrogen is taken to be inert.

In order to account for the strong interaction that exists between the chemistry and turbulence in nonpremixed combustion processes, the mean reaction rate, $\dot{w}_{n}$, is estimated using the eddy dissipation model (EDM) of Magnussen and Hjertager ${ }^{7}$

$$
\dot{\omega}_{\mathrm{F}}=-\mathcal{C}_{\mathrm{edm}} \frac{1}{\tau_{\mathrm{t}}} \rho \min \left(c_{\mathrm{F}}, \frac{c_{\mathrm{O}}}{s}\right), \quad \tau_{\mathrm{t}} \sim \frac{1}{\omega} .
$$

This model assumes that turbulence mixing limits the fuel burning and the fuel reaction rate is limited by the deficient species. The individual species mean reaction rate is then taken to be the minimum of the rates given by the finite-rate chemical kinetics (i.e., the law of mass action and Arrhenius reaction rates) and the EDM value. The latter is related to the turbulence mixing time and is estimated using the dissipation rate, $\omega$.

\section{Parallel AMR Algorithm}

\section{III.A. Finite Volume Scheme}

A finite volume scheme is employed to solve the system of Favre-averaged Navier-Stokes equations for a three-dimensional Cartesian coordinate frame. For three-dimensional flows, Equations (1)-(3), (5), (7), and (8) can be re-expressed using vector notation as

$$
\frac{\partial \mathbf{U}}{\partial t}+\frac{\partial}{\partial x}\left(\mathbf{F}-\mathbf{F}_{v}\right)+\frac{\partial}{\partial y}\left(\mathbf{G}-\mathbf{G}_{v}\right)+\frac{\partial}{\partial z}\left(\mathbf{H}-\mathbf{H}_{v}\right)=\mathbf{S},
$$

where $\mathbf{U}$ is the vector of conserved variables given by

$$
\mathbf{U}=\left[\rho, \rho v_{x}, \rho v_{y}, \rho v_{z}, \rho e, \rho k, \rho \omega, \rho c_{1}, \ldots, \rho c_{N}\right]^{\mathrm{T}},
$$

and the inviscid and viscous $x$-direction flux vectors, $\mathbf{F}$ and $\mathbf{F}_{v}$, can be written as

$$
\mathbf{F}=\left[\begin{array}{c}
\rho v_{x} \\
\rho v_{x}^{2}+p \\
\rho v_{x} v_{y} \\
\rho v_{x} v_{z} \\
(\rho e+p) v_{x} \\
\rho k v_{x} \\
\rho \omega v_{x} \\
\rho c_{1} v_{x} \\
\vdots \\
\rho c_{N} v_{x}
\end{array}\right], \mathbf{F}_{v}=\left[\begin{array}{c}
0 \\
\tau_{x x}+\lambda_{x x} \\
\tau_{x y}+\lambda_{x y} \\
\tau_{x z}+\lambda_{x z} \\
v_{x}\left(\tau_{x x}+\lambda_{x x}\right)+v_{y}\left(\tau_{x y}+\lambda_{x y}\right)+v_{z}\left(\tau_{x z}+\lambda_{x z}\right)-q_{x}-q_{t_{x}}+\left(\mu+\mu_{t} \sigma^{*}\right) \frac{\partial k}{\partial x} \\
\left(\mu+\mu_{t} \sigma^{*}\right) \frac{\partial k}{\partial x} \\
\left(\mu+\mu_{t} \sigma\right) \frac{\partial \omega}{\partial x} \\
-\mathcal{J}_{1 x}-\mathcal{J}_{t 1 x} \\
\vdots \\
-\mathcal{J}_{N x}-\mathcal{J}_{t N x}
\end{array}\right] .
$$




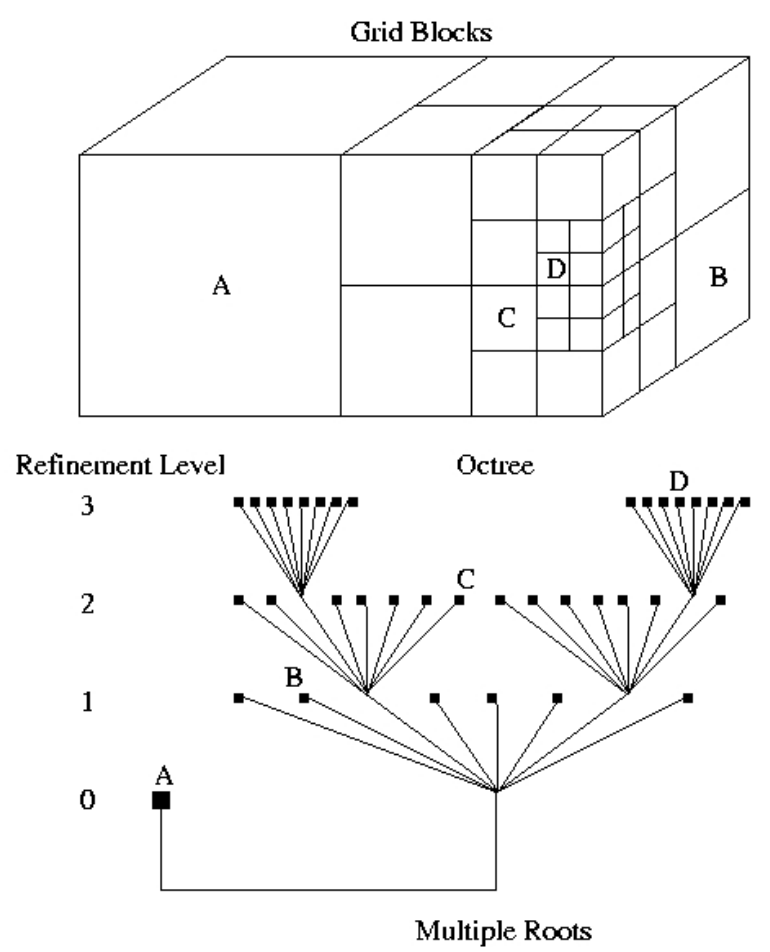

Figure 1. Multi-block hexahedral AMR mesh showing showing solution blocks at various levels of refinement and the corresponding octree data structure.

The $y$ - and $z$-direction flux vectors $\mathbf{G}, \mathbf{G}_{v}, \mathbf{H}$, and $\mathbf{H}_{v}$ have similar forms. The source vector, $\mathbf{S}$, appearing in Equation 21 contains terms related to the finite rate chemistry, body force due to gravity, and turbulence modelling and has the form

$$
\mathbf{S}=\left[0, f_{x}, f_{y}, f_{z}, \rho v_{z} g_{z}, \mathcal{P}-\beta^{*} \rho k \omega, \alpha \frac{\omega}{k} \mathcal{P}-\beta \rho \omega^{2}, \rho \dot{\omega}_{1}, \ldots, \rho \dot{\omega}_{N}\right]
$$

with

$$
\mathcal{P}=\lambda_{x x} \frac{\partial u}{\partial x}+\lambda_{x y}\left(\frac{\partial u}{\partial y}+\frac{\partial v}{\partial x}\right)+\lambda_{y y} \frac{\partial v}{\partial y}+\lambda_{x z}\left(\frac{\partial u}{\partial z}+\frac{\partial w}{\partial x}\right)+\lambda_{y z}\left(\frac{\partial v}{\partial z}+\frac{\partial w}{\partial y}\right)+\lambda_{z z} \frac{\partial w}{\partial z},
$$

and where $x, y$, and $z$ are the coordinates of the three dimensional Cartesian frame; $v_{x}, v_{y}$, and $v_{z}$ are the $x$, $y$, and $z$ velocity components; $f_{x}, f_{y}$, and $f_{z}$ are the components of body forces acting on the mixture; $q_{x}$, $q_{y}$, and $q_{z}$ are the $x, y$, and $z$ components of the heat flux; $\tau_{x x}, \tau_{x y}, \tau_{y y}, \tau_{x z}, \tau_{y z}$, and $\tau_{z z}$ are the components of the viscous fluid stresses; and $\lambda_{x x}, \lambda_{x y}, \lambda_{x z}, \lambda_{x z}, \lambda_{y z}$, and $\lambda_{z z}$ are the Reynolds stresses.

The system of Equation 21 is integrated over hexahedral cells of a structured body-fitted multi-block hexahedral mesh. The semi-discrete form of this finite-volume formulation applied to cell $(i, j, k)$ is given by

$$
\frac{d \mathbf{U}_{i, j, k}}{d t}=-\frac{1}{V_{i, j, k}} \sum_{\text {faces }, m} \overrightarrow{\mathbf{F}}_{i, j, k, m} \cdot \overrightarrow{\mathbf{n}}_{i, j, k, m} A_{i, j, k, m}+\mathbf{S}_{i, j, k}
$$

where $\overrightarrow{\mathbf{F}}=\left(\mathbf{F}-\mathbf{F}_{v}, \mathbf{G}-\mathbf{G}_{v}, \mathbf{H}-\mathbf{H}_{v}\right), V_{i, j, k}$ is the volume of cell $(i, j, k), A$ is the area of the cell face, and $\overrightarrow{\mathbf{n}}$ is the unit vector normal to the cell face. The inviscid (hyperbolic) components of the numerical flux at each cell face is evaluated using limited linear reconstruction ${ }^{45}$ and one of several Riemann-solver based flux functions. ${ }^{46-48}$ The viscous (elliptic) components of the cell face flux is evaluated by employing a hybrid average gradient-diamond path approach by Mathur and Murthy. ${ }^{49}$

For the time-invariant calculations performed as part of this study, a multi-stage time marching scheme is used to solve the coupled set of non-linear ordinary differential equations that arise from the finite-volume 


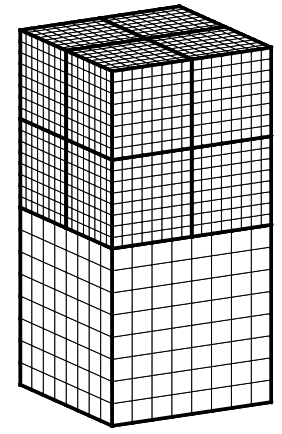

Figure 2. Examples of neighbouring refined and unrefined hexahedral solution blocks.

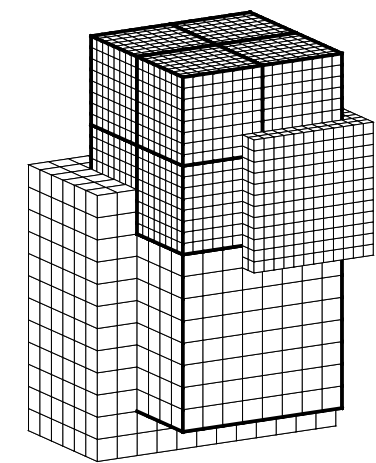

Figure 3. Examples of neighbouring refined and unrefined hexahedral solution blocks showing ghost cells used interblock sharing of solution information.

spatial discretization procedure. The time-marching scheme is based on the optimally-smoothing multistage time marching schemes developed by van Leer et al..$^{50}$ To cope with numerical stiffness, a semi-implicit treatment is used in the temporal discretization of the source terms associated with finite-rate chemistry, turbulence modelling, and body forces.

\section{III.B. Block-Based Adaptive Mesh Refinement}

Adaptive mesh refinement algorithms, which automatically adapt the mesh to the solution of the governing equations, can be very effective in treating problems with disparate length scales. They permit local mesh refinement and thereby minimize the number of computational cells required for a particular calculation. Following the approach developed by Groth et al. ${ }^{27,28}$ for computational magnetohydrodynamics, a flexible block-based hierarchical octree data structure, as illustrated in Figure 1, has been developed and is used in conjunction with the finite-volume scheme described above to facilitate automatic solution-directed mesh adaptation on multi-block body-fitted hexahedral mesh according to physics-based refinement criteria. Local refinement and coarsening of the mesh is carried out by division and merging of solution blocks, respectively.

Figure 2 shows two neighbouring blocks, one of which has undergone one level of refinement and one of which has not. Figure 3 depicts the "halo" or "ghost" cells that are used to facilitate communications between solution blocks. The proposed AMR approach allows for anisotropic mesh refinement and is well suited to parallel implementation via domain decomposition. Very efficient and highly scalable algorithms have been developed for the predictions of laminar combusting flows ${ }^{36}$ turbulent combusting flows, ${ }^{37,38}$ turbulent multi-phase rocket motor core flows, ${ }^{51}$ micro-scale flows, ${ }^{52}$ and compressible flows with high-order scheme, ${ }^{53}$ all in two space dimensions.

\section{III.C. Domain Decomposition and Parallel Implementation}

A parallel implementation of the block-based AMR scheme has been developed using the $\mathrm{C}++$ programming language and the MPI (message passing interface) library. ${ }^{54}$ A domain decomposition procedure is used where the solution blocks making up the computational mesh are distributed equally among available 


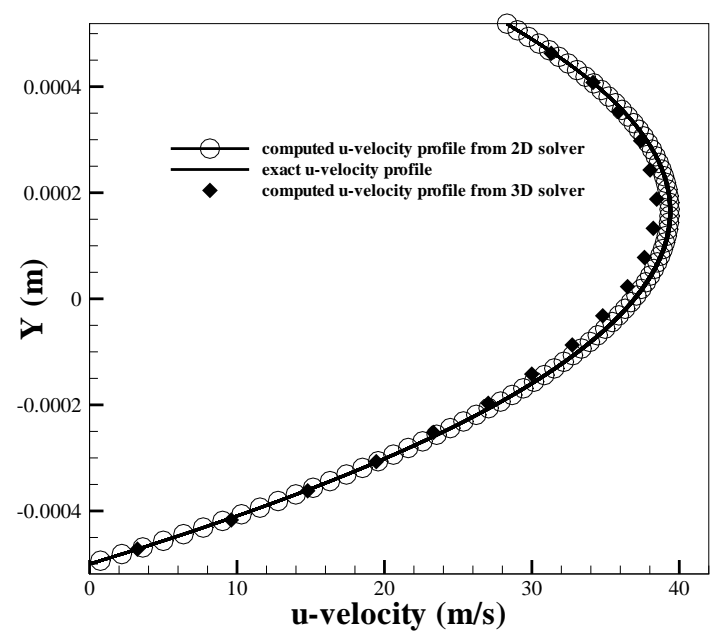

Figure 5. Comparison of the predicted axial velocity profiles for non-reacting laminar Couette flow to exact analytical solution.
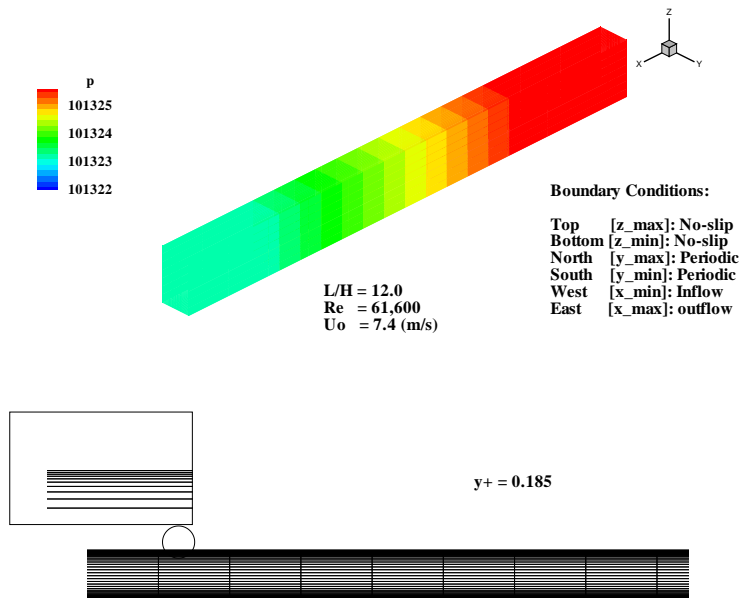

Figure 6. Channel flow geometry and stretched hexahedral computational mesh used in $\mathrm{Re}=61,600$ turbulent flow channel flow calculation.

processors, with more than one block permitted per processor. A Morton ordering space filling curve, as shown in Figure ??, is used to provide nearest-neighbour ordering of the solution blocks in the multi-block hexahedral AMR mesh for more efficient load balancing. ${ }^{55}$

The parallel implementation has been carried out on a parallel cluster of 4-way Hewlett-Packard ES40, ES45, and Integrity rx4640 servers with a total of 244 Alpha and Itanium 2 processors. A low-latency Myrinet network and switch is used to interconnect the servers in the cluster.

\section{Numerical Validation}

For two-dimensional planar and axisymmetric geometry, the proposed parallel AMR scheme has been previously validated for non-reacting flows and both laminar diffusion flames ${ }^{36}$ and turbulent combusting flows. ${ }^{37,38}$ Validation of the proposed three-dimensional algorithm for multi-block hexahedral mesh is considered herein for non-reacting laminar and turbulent flows. The computation of non-reacting laminar Couette flow in a channel with a moving wall is considered in order to validate the implementation of the spatial discretization scheme for the viscous operators. The numerical results are compared to the exact analytical solution for this case. Furthermore, the validation of the proposed algorithm for non-reacting turbulent flows is performed by comparing numerical results to the experimental data of Laufer ${ }^{56,57}$ for non-reacting fullydeveloped turbulent flows in both a duct and a pipe. This second set of the comparisons provides partial validation of the implementation of the two-equation turbulence model and near-wall sublayer treatments. The results of these comparisons are summarized in the following subsections.

\section{IV.A. Non-Reacting Laminar Couette Flow}

The computation of non-reacting laminar Couette flow in a channel with a moving upper wall velocity of $29.4 \mathrm{~m} / \mathrm{s}$ and a favourable pressure gradient of $d p / d x=-3,177 \mathrm{~Pa} / \mathrm{m}$ was investigated and compared to the analytic solution. A graph of the predicted velocity profile is shown in Figure 4 and compared to the exact analytic solution and predicted velocity profile of the two-dimensional version of the proposed algorithm reported in earlier studies. ${ }^{37,38}$ For this nearly incompressible isothermal flow, it can be seen that the predicted results of the three-dimensional algorithm match well with the analytical solution and the two-dimensional predictions. This verifies the implementation of the numerical viscous flux operators.

\section{IV.B. Non-Reacting Turbulent Channel and Pipe Flows}

The validation of the solver for non-reacting turbulent flows was performed by comparing numerical results to the experimental data of Laufer ${ }^{56,57}$ for non-reacting, fully-developed turbulent flow in a rectangular 


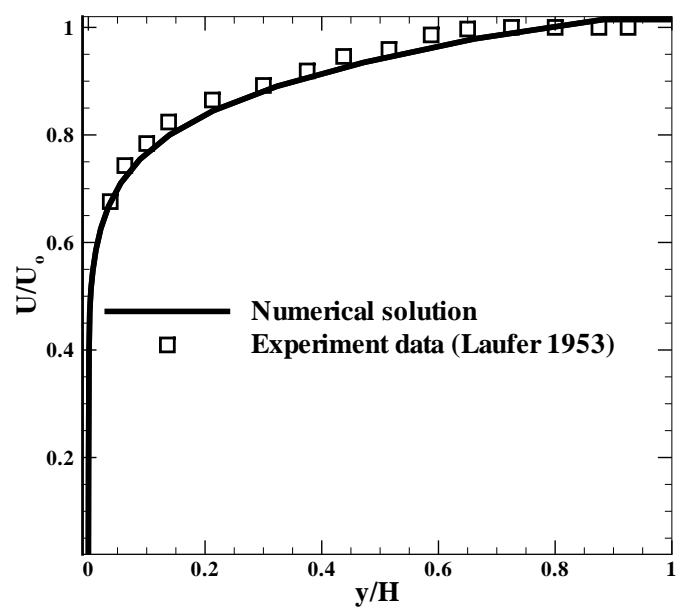

Figure 7. Comparison of the predicted cross-channel mean axial velocity profile for $\mathrm{Re}=61,600$ turbulent channel flow to the experimental data of Laufer. ${ }^{57}$

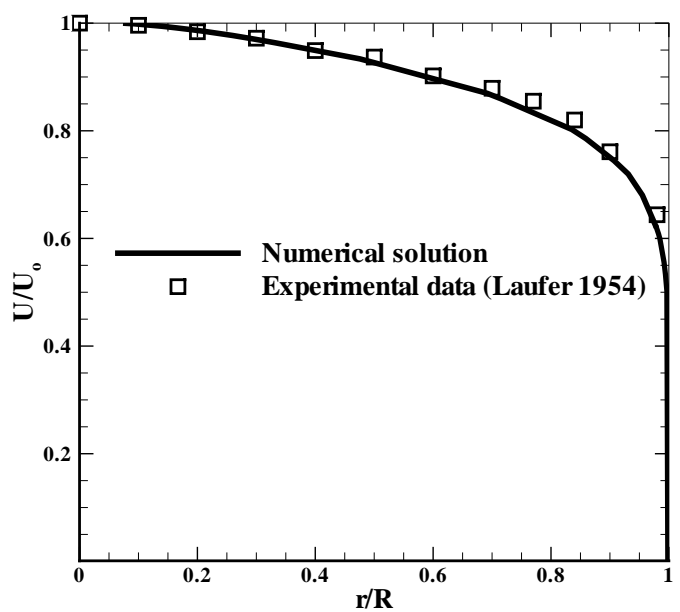

Figure 9. Comparison of the predicted radial profile of the mean axial velocity for $\mathrm{Re}=500,000$ turbulent pipe flow to the experimental data of Laufer. ${ }^{56}$

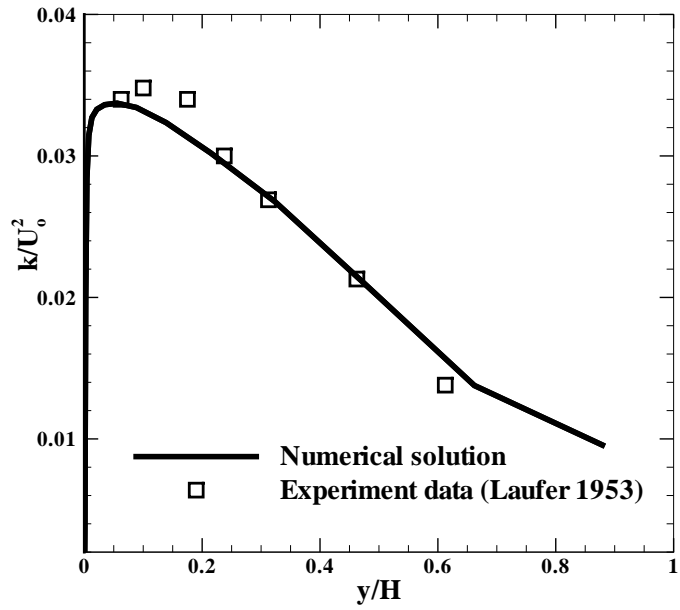

Figure 8. Comparison of the predicted cross-channel turbulent kinetic energy profile for $\mathrm{Re}=61,600$ turbulent channel flow to the experimental data of Laufer. ${ }^{57}$

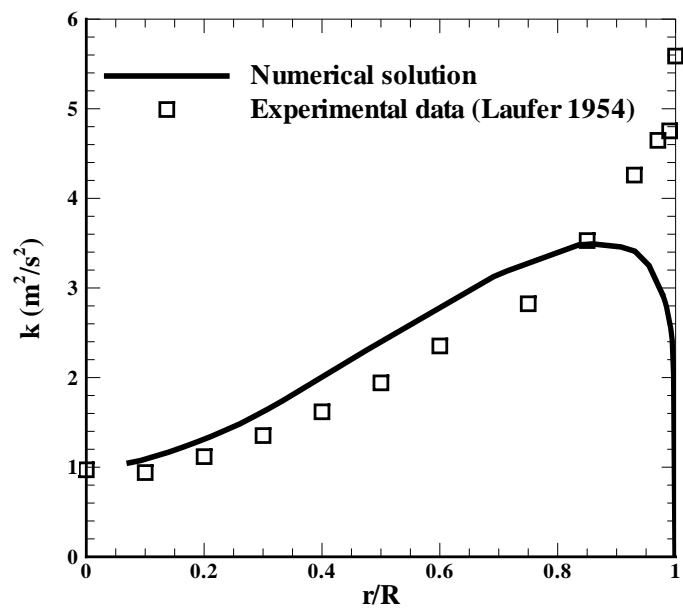

Figure 10. Comparison of the predicted radial profile of the turbulent kinetic energy for $\mathbf{R e}=500,000$ turbulent pipe flow to the experimental data of Laufer. ${ }^{56}$

channel and a circular cross-section pipe. The working gas was air in both cases and, for the channel flow, the width of the channel was $0.127 \mathrm{~m}$ and the Reynolds number based on the centre-line velocity, $u_{o}=7.4$ $\mathrm{m} / \mathrm{s}$, was $\operatorname{Re}=61,600$. For the pipe flow, the radius of the pipe was 0.123 and the Reynolds number based on the centre-line velocity, $u_{o}=30.48 \mathrm{~m} / \mathrm{s}$, was $\operatorname{Re}=500,000$. Figure 5 depicts the channel flow geometry and boundary condition setup.

Predicted numerical solutions obtained using the proposed parallel AMR scheme for both the turbulent channel and pipe flows are given in Figures 6-9. The numerical predictions were obtained by integrating the transport equations for the two-equation turbulent model through the laminar sublayer directly to the channel and pipe wall. The calculations were performed using 32 cells in the cross-channel and radial directions for both cases and near-wall stretching of the mesh was applied such that there were 2-3 of computational cells within the laminar sublayer. The first cell off the wall was located at $y^{+}=0.07$ and $y^{+}=0.0175$ for channel and pipe flows, respectively. The predictions of the mean axial velocity and turbulent kinetic energy are compared to the experimental data of Laufer ${ }^{56,57}$ in the figures. In general, good agreement between the experimental data and numerical results is observed and it is evident that the $k-\omega$ model is able to reproduce the characteristic features of these two fully-developed non-reacting turbulent flows. 


\section{Numerical Results for Bluff-Body Burner}

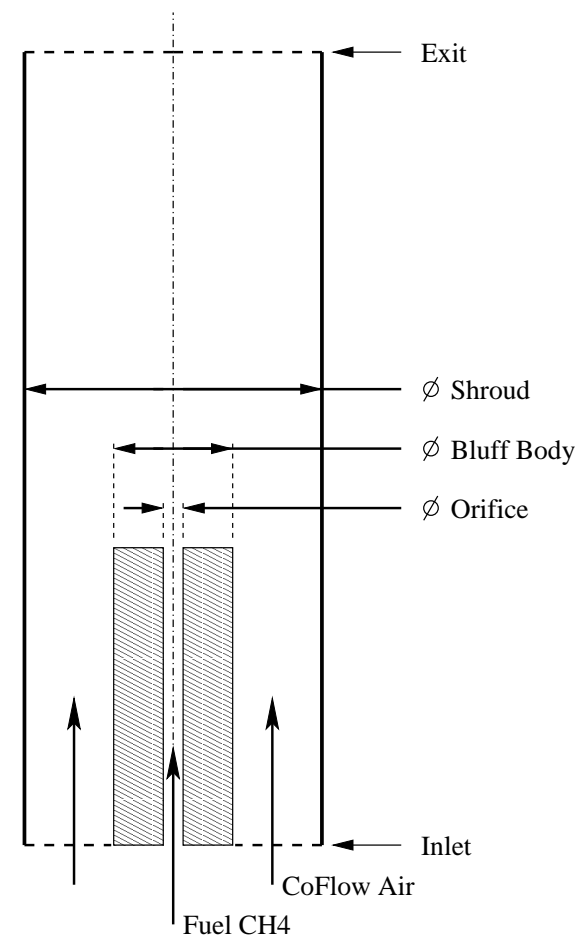

Figure 11. Schematic of Sydney bluffbody burner geometry.

hot-flow cases.
The International Workshops on Measurement and Computation of Turbulent Non-Premixed Flames (TNF) have lead to the establishment of an Internet library of well-documented turbulent nonpremixed flames that are appropriate for combustion model validation. ${ }^{58}$ The proposed parallel AMR method has been applied to the solution of one non-reacting flow case and one reacting flow case associated with the Sydney bluff-body burner configuration that forms part of this experimental database. The Sydney bluff-body burner have been investigated and/or used for validation purposes in several recent studies (e.g., Dally et al. ${ }^{59-64}$ and Turpin and Troyes ${ }^{65}$ ).

The geometry of the bluff-body burner is shown in Figure 10. The bluff-body has a diameter of $D_{b}=50 \mathrm{~mm}$ and is located in a co-axial flow of air. Various gases can be injected through an orifice of diameter $3.6 \mathrm{~mm}$ at the base of the cylindrical bluff-body. For cold-flow cases, air is injected, for cold flows where fuel mixing is of interest, gaseous fuels such as ethylene $\left(\mathrm{C}_{2} \mathrm{H}_{4}\right)$ are injected, and for hot-flow cases, gaseous fuels and fuel mixture such as methane $\left(\mathrm{CH}_{4}\right)$ are injected. In the hot-flow cases, the bluff-body stabilized flames have a recirculation zone close to the base of bluff body that produces a large and complex turbulent field and causes intense mixing between the reactants and the combustion products. The stabilization mechanisms resemble that of industrial combustors and yet the boundary conditions in these flames are simple and well-defined, making it well suited for investigating in great detail the capabilities of turbulent combustion models for non-premixed diffusion flames. In what follows, the predicted flow fields for the bluff-body burner are analyzed and compared with available experimental data for both cold- and

\section{V.A. Non-Reacting Cold Flow}

In the cold non-reacting bluff-body burner flow case considered herein, air is injected through the orifice at the base of the cylindrical bluff body with a temperature of $300 \mathrm{~K}$ and velocity of $61 \mathrm{~m} / \mathrm{s}$. The velocity and temperature of the co-flowing air is $20 \mathrm{~m} / \mathrm{s}$ and $300 \mathrm{~K}$, respectively. The Reynolds and Mach numbers based on the high-speed jet are $\mathrm{Re}=193,000$ and $\mathrm{Ma}=0.18$.

A cylindrical-shaped computational domain is used with a diameter of $3 D_{b}$ and a length of $6 D_{b}$, such that the influence of the far-field domain boundaries are minimized. Flow parameters such as velocity field

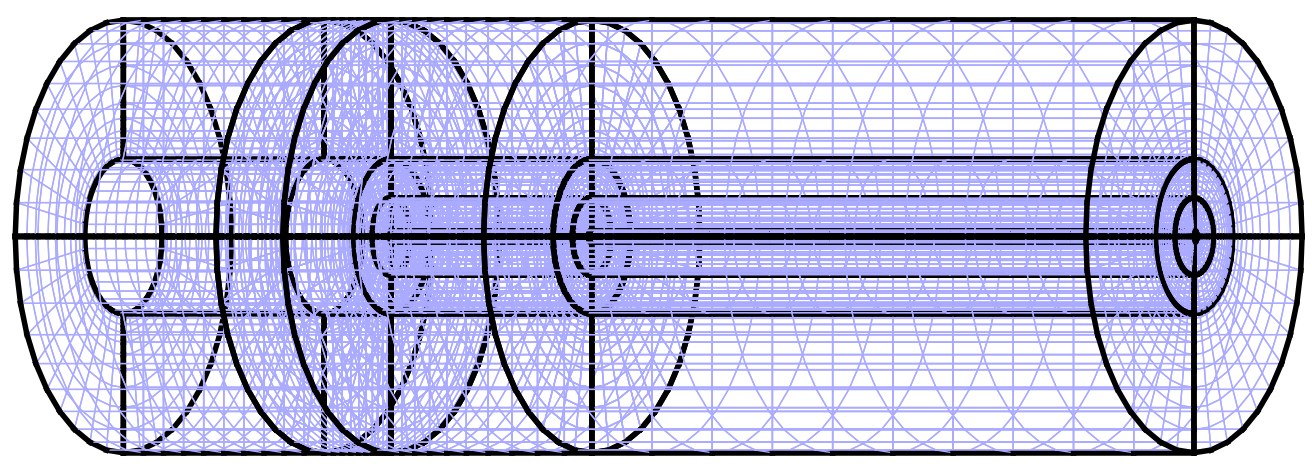

Figure 12. Computational mesh used in prediction of bluff-body burner cold flow consisting of $4210 \times 10 \times 10-c e l l$ solution blocks and 42,000 cells. 


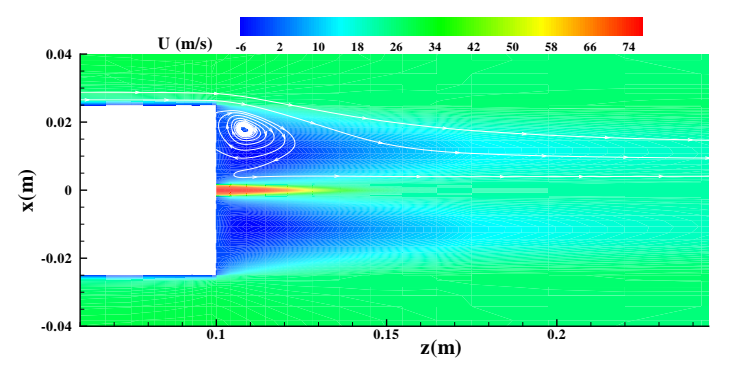

Figure 13. Predicted mean axial velocity field in the $y=$ $0 x z$-plane of the bluff-body for non-reacting bluff-body burner with air jet.

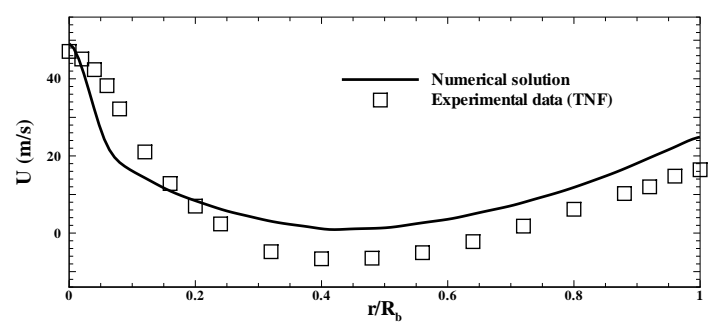

(a) $z / D_{b}=0.6$

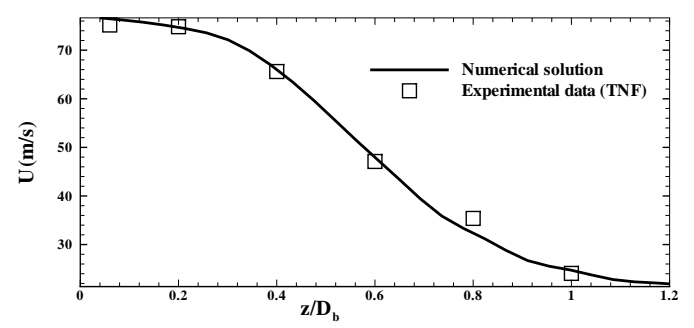

Figure 14. Comparison of predicted and measured onaxis axial profiles of mean axial velocity component downstream from the base of the bluff-body burner for nonreacting flow with air jet.

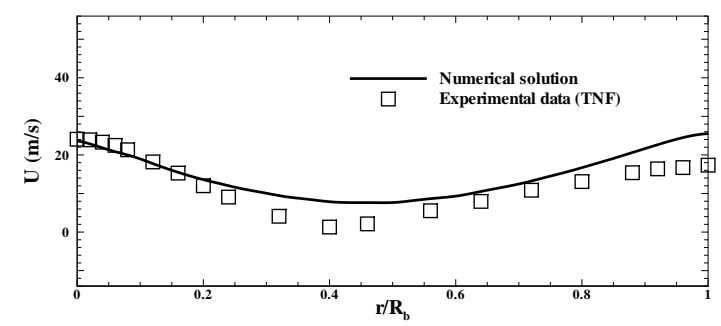

(b) $z / D_{b}=1.0$

Figure 15. Predicted mean axial velocity radial profiles at $z / D_{b}=0.6$ and $z / D_{b}=1.0$ downstream from the base of the bluff-body burner for non-reacting flow with air jet.

and mixture fractions at the inflow plane are specified using Dirichlet-type boundary conditions. At the outflow plane and circumferential surface, Neumann-type conditions are applied with the gradients of the flow solution quantities set to zero. A computational mesh consisting of $4210 \times 10 \times 10$-cell solution blocks and 42,000 cells was used. The mesh is given Figure 11. The cells of the computational grid are clustered in the regions of strong gradients of the mean mixture solution quantities, outer and inner shear layers, and recirculation zones near the walls. The mesh resolution typically provides for off-wall spacings for the first cells nearest the wall in the range of $0.7<y^{+}<1.2$.

Figure 12 shows colour contours of the predicted mean axial velocity with superimposed mean streamlines in $y=0 x z$-plane obtained from the fully three-dimensional solution of the bluff-body burner cold-flow case. The figure reveals the formation of a vortex structure in the re-circulation zone which are important in controlling fuel/oxidizer mixing. The calculations indicate that the size of re-circulation zone is slightly less than the experimentally observed value. This is very similar to previous findings of the authors when comparing the axisymmetric predictions to the experimental data. ${ }^{37,38}$

Figure 13 depicts the axial (centre-line) profile of the mean axial velocity component. In addition, Figures 14(a) and 14(b) show comparisons between the predicted and measured radial profiles of the mean axial velocity component at $z / D_{b}=0.6$ and $z / D_{b}=1.0$ downstream from the base of the bluff-body burner. For each profile shown, good agreement between the numerical predictions and experiment can be observed.

Figures 15(a)-16(f) show comparisons of the predicted and measured values of the root mean square (RMS) fluctuations of the velocity components. The computed and measured specific Reynold stress $\overline{u^{\prime} v^{\prime}}$ are also compared in Figures 17(a)-17(f). The measured RMS of fluctuations of the velocity components have peak values at $x / R_{b}=1.0$ for $z / D_{b}=0.06$; this corresponds to the shear layer between the co-flow and the bluff-body wake. The numerical predictions also seem to capture this feature. The computed specific Reynold stresses, $\overline{u^{\prime} v^{\prime}}$, are over-predicted in the relatively high mean-velocity regions, but agree with the measured data in the low mean-velocity regions. It can be seen that there are under- and/or over-predicted regions close to the fuel inlet and the solid wall boundaries. These regions are either parts of or close to the recirculation zone. Recirculation zones with complex turbulent structure are very sensitive to standard RANS closures. A variety of RANS simulations have addressed the sensitivity of the results to the turbulence model and/or combustion models. ${ }^{37,38,63,66-68}$ The overall agreement between the predicted results from the current study and the experimental data are reasonable. Findings are comparable to other work in the 


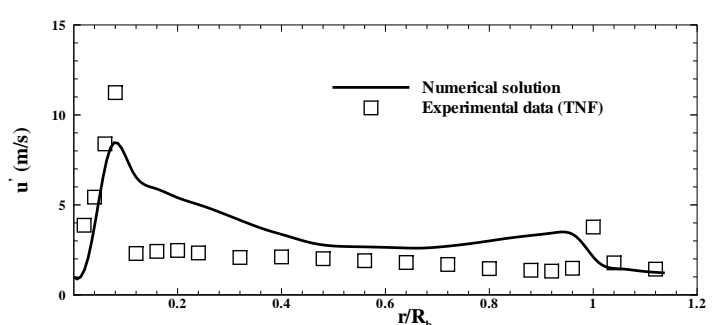

(a) $z / D_{b}=0.06$

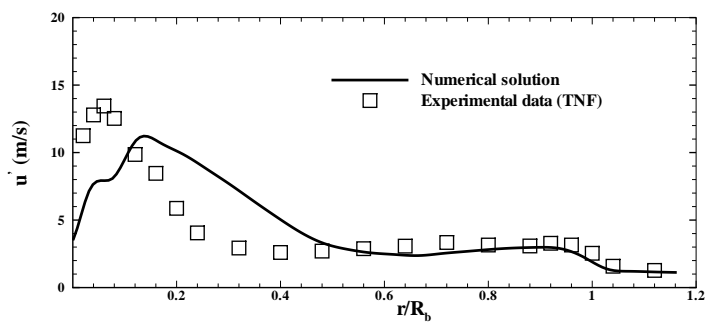

(c) $z / D_{b}=0.4$

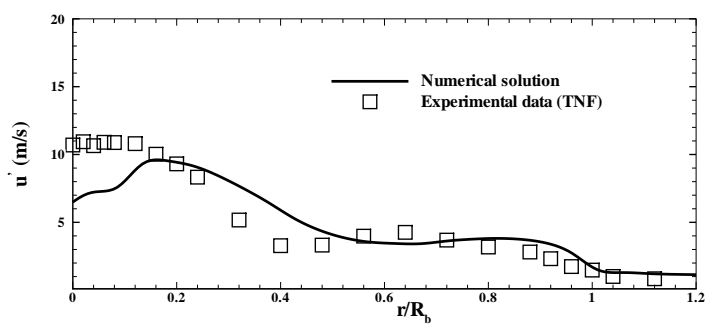

(e) $z / D_{b}=0.8$

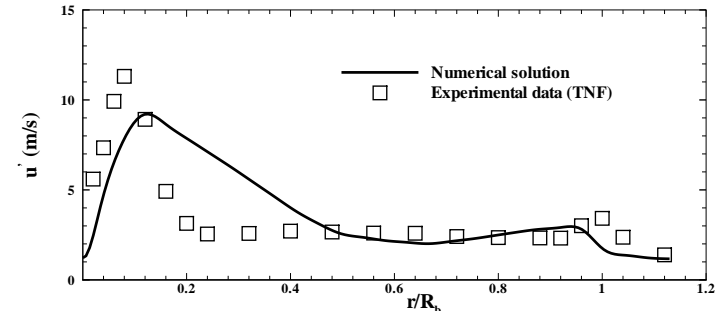

(b) $z / D_{b}=0.2$

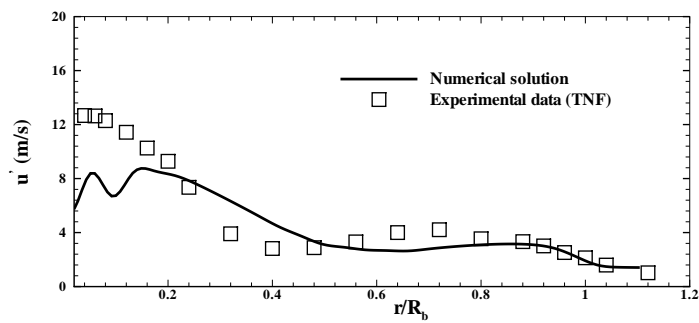

(d) $z / D_{b}=0.6$

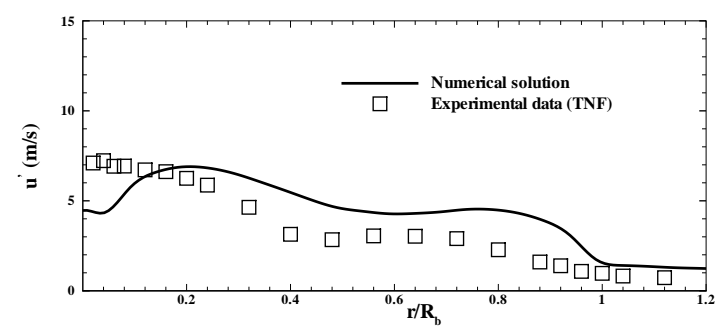

(f) $z / D_{b}=1.0$

Figure 16. Comparison of predicted and measured radial profiles of $\sqrt{\overline{{u^{\prime 2}}^{2}}}$ at various locations downstream from the base of the bluff-body burner for non-reacting flow with air jet.

literature. $63,65,67$

\section{V.B. Reacting Hot Flow}

For the reacting case, methane is injected through the orifice at the base of the cylindrical bluff body with a temperature of $300 \mathrm{~K}$. The flow geometry and boundary conditions for the reacting case are essentially the same as those for the non-reacting case, except that the velocities of the co-flowing air and methane fuel are $25 \mathrm{~m} / \mathrm{s}$ and $108 \mathrm{~m} / \mathrm{s}$, respectively. The Reynolds and Mach numbers of the methane jet are $R e=315,000$ and $\mathrm{Ma}=0.24$. The reactive flow field predictions have been performed on a mesh consisting of $4216 \times 16 \times 16$-cell solution blocks with a total of 172,032 cells. Similar to the non-reacting case, the mesh resolution provides for a typical off-wall spacing of the first computational cells nearest the wall to be in the range $0.7<y^{+}<1.2$.

Figures 18(a) and 18(b) provide the predicted distributions of mean temperature and mean mass fraction of $\mathrm{CO}_{2}$ on the $y=0 x z$-plane for this turbulent non-premixed flame. The predicted flame structure is generally in agreement with the experimentally observed structure. The flame is quite elongated and three zones can be identified: the re-circulation, neck, and jet-like propagation zones. A vortex structure is formed in the re-circulation zone and acts to stabilize the flame. The predicted maximum flame temperature is about $2112 \mathrm{~K}$, which is close to the value of $2180 \mathrm{~K}$ observed in the author's previous axisymmetric studies of this bluff-body hot-flow case. ${ }^{37,38}$

Predicted radial profiles of the mean temperature and mean mass fraction of $\mathrm{CO}_{2}$ at a location of $z / D_{b}=1.92$ downstream from the base of the bluff-body are shown in Figures 19(a) and 19(b) and compared with experimental results. The comparisons shown in the figure indicate that both the predicted mean temperature and mass fraction of $\mathrm{CO}_{2}$ are somewhat over-predicted. However, it is felt that the agreement 


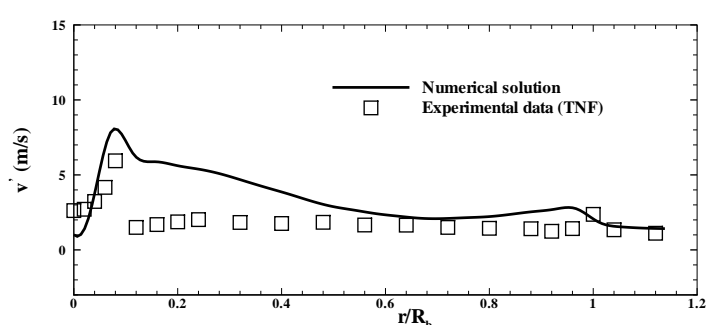

(a) $z / D_{b}=0.06$

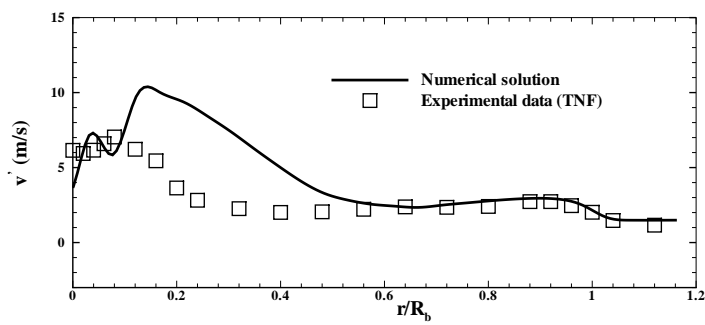

(c) $z / D_{b}=0.4$

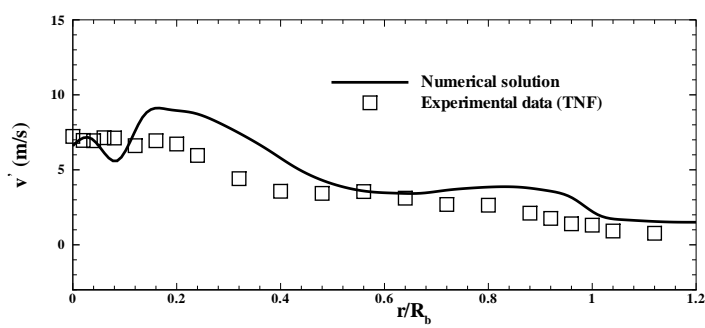

(e) $z / D_{b}=0.8$

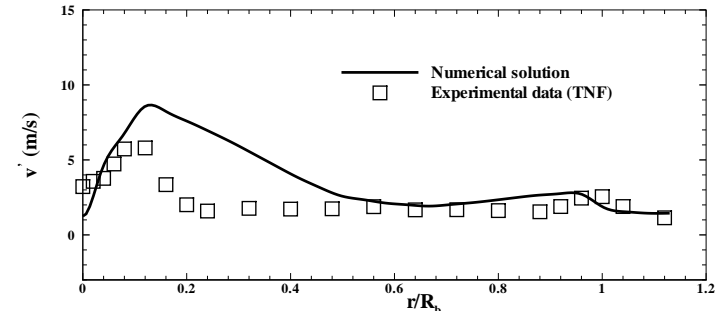

(b) $z / D_{b}=0.2$

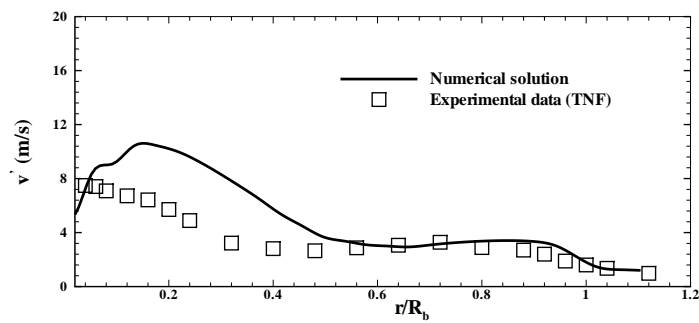

(d) $z / D_{b}=0.6$

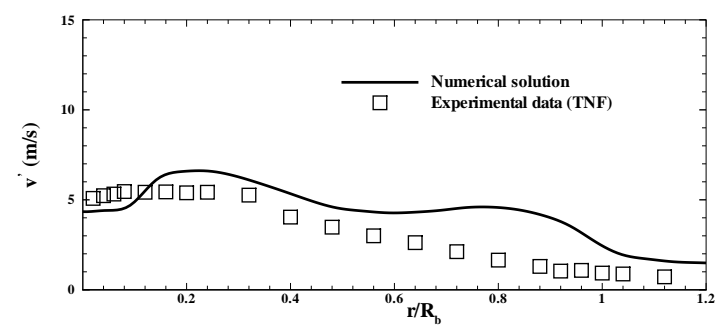

(f) $z / D_{b}=1.0$

Figure 17. Comparison of predicted and measured radial profiles of $\sqrt{\overline{v^{\prime 2}}}$ at various locations downstream from the base of the bluff-body burner for non-reacting flow with air jet.

with the experimental values is reasonable considering the limitations of the simplified reduced chemical kinetics scheme and turbulence/chemistry interaction model used herein, as well as the fact that radiation transport is not taken into account in the simulation. Note that radiation effects may influence the predicted temperature for this case; however, Merci et al. argues that the flame is unconfined and very little soot is formed and so radiation effects should be small. ${ }^{67,69}$

\section{Parallel Performance}

Estimates of the parallel performance and scalability of the proposed solution algorithm are shown in Figure 20 for the Sydney bluff-body burner flame problem described in the previous section. For this fixed size problem, a multi-block hexahedral computational mesh consisting of 21,504 cells $(8 \times 8 \times 8$ blocks $)$ and 42 solution blocks was used and the parallel performance measures for the algorithm for up to 42 processors is depicted in the figure. Both the scaled parallel speed-up,

$$
S_{p}=\frac{t_{1}}{t_{p}} p
$$

and the scaled parallel efficiency,

$$
E_{p}=\frac{S_{p}}{p},
$$

are shown for the problem as a function of the number of processors, $p$, where $t_{p}$ is the total processor time required to solve the problem using $p$ processors and $t_{1}$ is the processor time required to solve the problem 


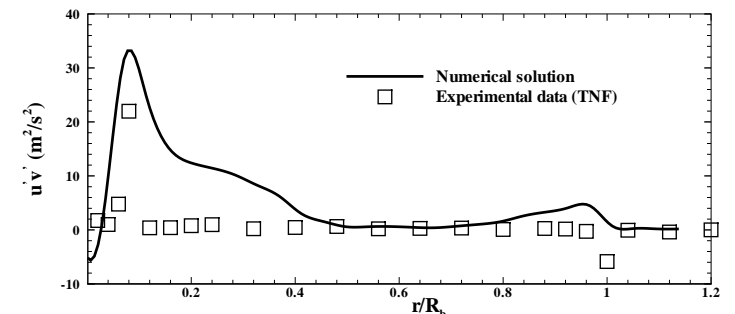

(a) $z / D_{b}=0.06$

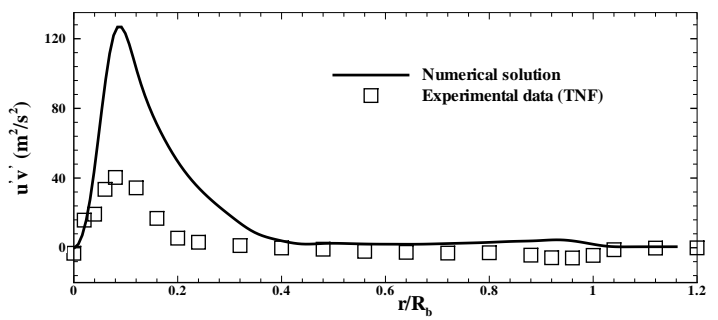

(c) $z / D_{b}=0.4$

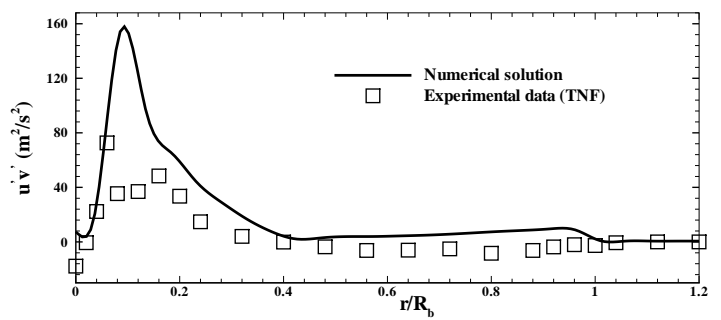

(e) $z / D_{b}=0.8$

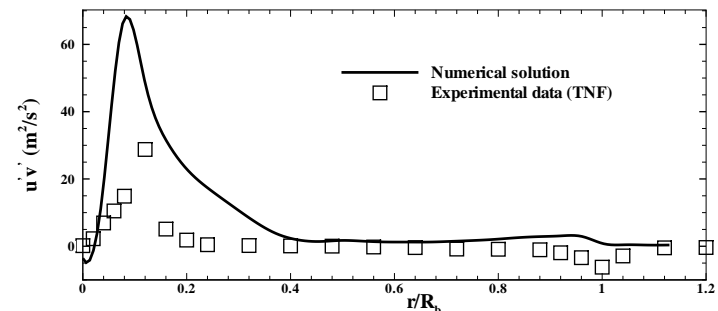

(b) $z / D_{b}=0.2$

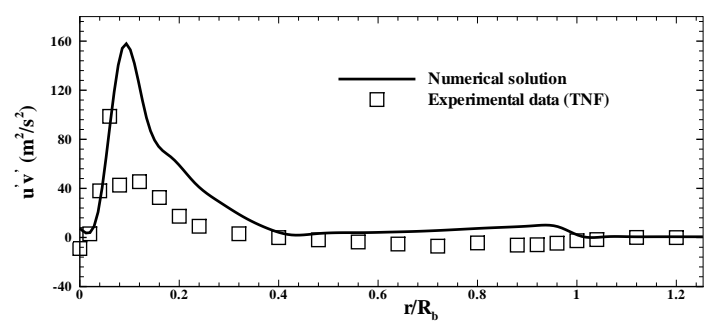

(d) $z / D_{b}=0.6$

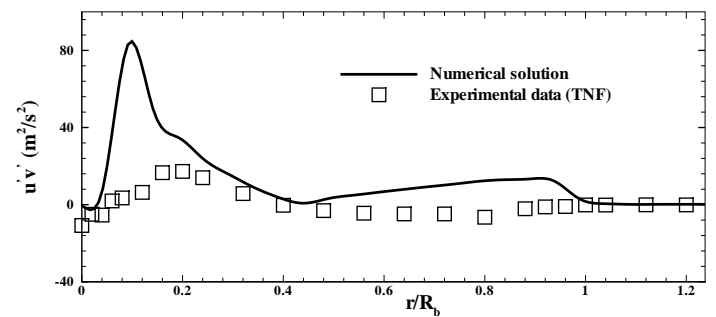

(f) $z / D_{b}=1.0$

Figure 18. Comparison of predicted and measured radial profiles of $\overline{u^{\prime} v^{\prime}}$ at various locations downstream from the base of the bluff-body burner for non-reacting flow with air jet.

using a single processor. Figure 20 indicates that the parallel speed-up is nearly linear and is about $98 \%$ efficient for up to 42 processors.

\section{Concluding Remarks}

A highly parallelized AMR scheme has been described for turbulent non-premixed combusting flows. The combination of a block-based AMR strategy and parallel implementation has resulted in a highly scalable computational tool for simulating three-dimensional non-premixed turbulent combusting flows. Predictions of the proposed parallel solution were obtained for a bluff-body stabilized turbulent diffusion flame and the numerical solutions were compared to available experimental data. For the non-reacting cold-flow case, the numerical results were generally found to be in good agreement with the experimental data, as well as comparable to other similar numerical results reported in the literature. For the reacting hot-flow case, the predicted mean temperature and mean mass fractions of some major species are somewhat over-predicted as compared to experimental. However, given the complexity of the combusting flow field and the limitations of the reduced chemical reaction mechanism and simplified turbulence/chemistry interaction model adopted here, the preliminary results are quite encouraging and indicate the potential of the algorithm for predicting complex combusting flows. Future work will include the investigation of Newton-Krylov-Schwarz strategies $^{70-72}$ in an effort to improve the efficiency of the time integration procedure while maintaining high parallel efficiency and the inclusion of more sophisticated combustion modelling beyond the simplified models used herein. 


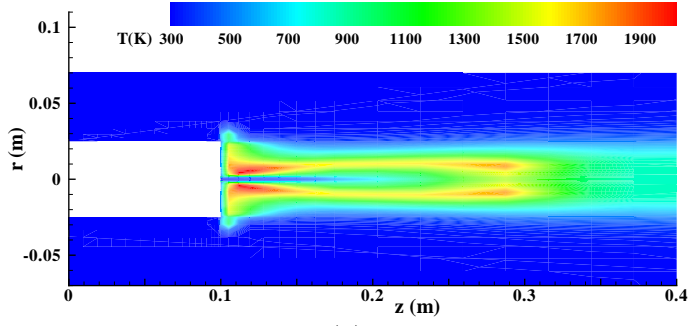

(a) $\mathrm{T}$

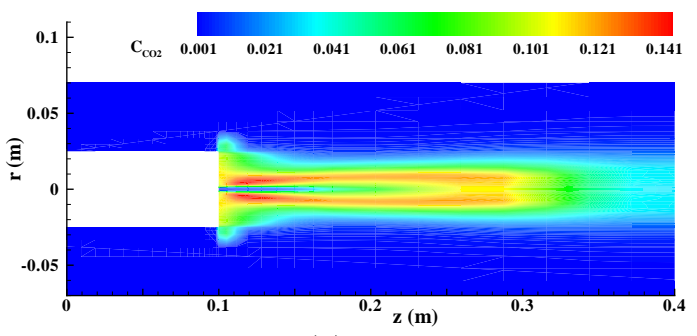

(b) $\mathrm{CO}_{2}$

Figure 19. Predicted mean temperature and mean $\mathrm{CO}_{2}$ mass fraction distributions in the $y=0 x z$-plane of the bluff-body burner for reacting flow with $\mathrm{CH}_{4}$ jet.

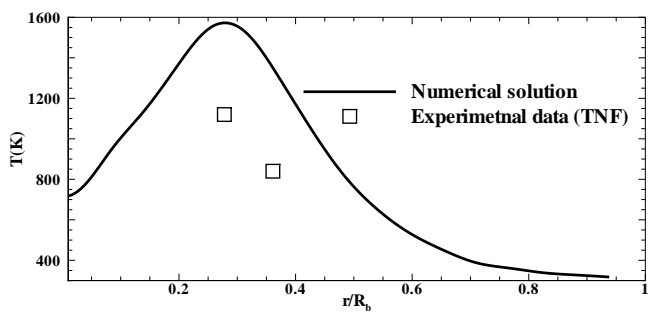

(a) $\mathrm{T}$

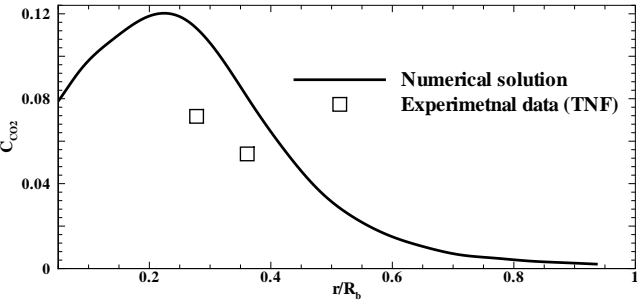

(b) $\mathrm{CO}_{2}$

Figure 20. Predicted mean temperature field and mean $\mathrm{CO}_{2}$ mass fraction radial profiles at $z / D_{b}=1.92$ downstream from the base of the bluff-body burner for reacting flow with $\mathrm{CH}_{4}$ jet.

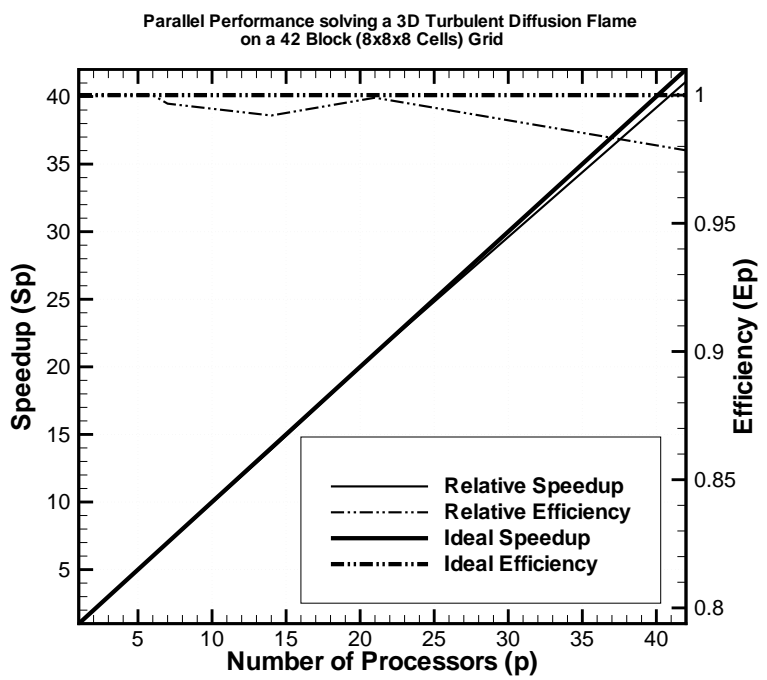

Figure 21. Relative parallel speed-up and efficiency for computation of Sydney bluff-body burner flame problem with a fixed-size turbulent diffusion flame problem with a multi-block hexahedral computational mesh consisting of 21,504 cells $(8 \times 8 \times 8$ blocks $)$ and 42 solution blocks using up to 42 processors.

\section{Acknowledgments}

This research was partially supported by a Collaborative Research Opportunities Grant from the Natural Sciences and Engineering Research Council of Canada. Funding for the parallel computing facility used to perform the computations described herein was obtained from the Canadian Foundation for Innovation and Ontario Innovation Trust (CFI Project No. 2169). The authors would like to thank Stephen Guzik for contributing insight and a methodology for determining hexahedral solution block connectivity. 


\section{References}

${ }^{1}$ Vervisch, L., Hauguel, R., Domingo, P., and Rullaud, M., "Three Facets of Turbulent Combustion Modelling: DNS of Premixed V-Flame, LES of Lifted Nonpremixed Flame and RANS of Jet-Flame," Journal of Turbulence, Vol. 5, 2004, pp. 1-36.

${ }^{2}$ Cant, S., "High-Performance Computing in Computational Fluid Dynamics: Progress and Challenges," Philosophical Transactions: Mathematical, Physical and Engineering Sciences, Vol. 360, No. 1795, 2002, pp. 1211-1225.

${ }^{3}$ Vervisch, L., "DNS and LES of Turbulent Combustion," Computational Fluid Dynamics in Chemical Reaction Engineering IV, June 19-24, 2005, Barga, Italy.

${ }^{4}$ Piomelli, U., "Large-Eddy Simulation: Achievements and Challenges," Prog. Aerospace Sci., Vol. 35, 1999, pp. 335-362. 2000.

${ }^{5}$ Davis, S., Freund, L., Leibovich, S., and Tvergaard, V., editors, Turbulent Combustion, Cambridge University Press,

${ }^{6}$ Veynante, D. and Poinsot, T., "Large Eddy Simulation of Combustion Instabilities in Turbulent Premixed Burners," Annual Research Briefs 253-274, Center for Turbulence Research, NASA Ames/Stanford Univ., 2000.

${ }^{7}$ Poinsot, T. and Veynante, D., Theoretical and Numerical Combustion, R.T. Edwards Inc., 2001.

${ }^{8}$ Selle, L., Lartigue, G., Poinsot, T., Krebs, P. K. W., and Veynante, D., "Large-Eddy Simulation of Turbulent Combustion for Gas Turbines with Reduced Chemistry," Proceedings of the Summer Program, 2002, pp. 333 - 344.

${ }^{9}$ Fox, R. O., editor, Computational Models for Turbulent Reacting Flows, Cambridge University Press, Cambridge, 2003.

${ }^{10}$ Berger, M. J., "Adaptive Mesh Refinement for Hyperbolic Partial Differential Equations," Journal of Computational Physics, Vol. 53, 1984, pp. 484-512.

${ }^{11}$ Berger, M. J. and Colella, P., "Local Adaptive Mesh Refinement for Shock Hydrodynamics," Journal of Computational Physics, Vol. 82, 1989, pp. 67-84.

${ }^{12}$ Thompson, M. and Ferziger, J., "An Adaptive Multigrid Technique for the Incompressible Navier-Stokes Equations," Journal of Computational Physics, Vol. 82, No. 1, 1989, pp. 94-121.

${ }^{13}$ Quirk, J. J., An Adaptive Grid Algorithm for Computational Shock Hydrodynamics, Ph.D. thesis, Cranfield Institute of Technology, January 1991.

${ }^{14}$ Powell, K. G., Roe, P. L., and Quirk, J., "Adaptive-Mesh Algorithms for Computational Fluid Dynamics," Algorithmic Trends in Computational Fluid Dynmaics, edited by M. Y. Hussaini, A. Kumar, and M. D. Salas, Springer-Verlag, New York, 1993, pp. 303-337.

${ }^{15}$ De Zeeuw, D. and Powell, K. G., "An Adaptively Refined Cartesian Mesh Solver for the Euler Equations," Journal of Computational Physics, Vol. 104, 1993, pp. 56-68.

${ }^{16}$ Quirk, J. J. and Hanebutte, U. R., "A Parallel Adaptive Mesh Refinement Algorithm," Report 93-63, ICASE, August 1993.

${ }^{17}$ Berger, M. J. and Saltzman, J. S., "AMR on the CM-2," Applied Numerical Mathematics, Vol. 14, 1994, pp. $239-253$.

${ }^{18}$ Almgren, A. S., Buttke, T., and Colella, P., "A Fast Adaptive Vortex Method in Three Dimensions," Journal of Computational Physics, Vol. 113, 1994, pp. 177-200.

${ }^{19}$ Bell, J., Berger, M., Saltzman, J., and Welcome, M., "A Three-Dimensional Adaptive Mesh Refinement for Hyperbolic Conservation Laws," SIAM Journal on Scientific Computing, Vol. 15, 1994, pp. 127-138.

${ }^{20}$ Pember, R. B., Bell, J. B., Colella, P., Crutchfield, W. Y., and Welcome, M. L., "An Adaptive Cartesian Grid Method for Unsteady Compressible Flow in Irregular Regions," Journal of Computational Physics, Vol. 120, 1995, pp. $278-304$.

${ }^{21}$ Aftomis, M. J., Berger, M. J., and Melton, J. E., "Robust and Efficient Cartesian Mesh Generation for Component-Base Geometry," AIAA Journal, Vol. 36, No. 6, 1998, pp. 952-960.

${ }^{22}$ Almgren, A. S., Bell, J. B., Colella, P., Howell, L. H., and Welcome, M. J., "A Conservative Adaptive Projection Method for the Variable Density Incompressible Navier-Stokes Equations," Journal of Computational Physics, Vol. 142, 1998, pp. 1-46.

${ }^{23}$ Pember, R. B., Howell, L., Bell, J., Colella, P., Crutchfield, W. Y., Fiveland, M. A., and Jessee, J., "An Adaptive Projection Method for Unsteady, Low Mach Number Combustion," Combustion Science and Technology, Vol. 140, 1998, pp. $123-168$.

${ }^{24}$ Jessee, J., Fiveland, W., Howell, L., Colella, P., and Pember, R., "An Adaptive Mesh Refinement Algorithm for the Radiative Transport Equation," Journal of Computational Physics, Vol. 139, No. 2, 1998, pp. 380-398.

${ }^{25}$ Colella, P., Dorr, M., and Wake, D., "Numerical Solution of Plasma-Fluid Equations Using Locally Refined Grids," Journal of Computational Physics, Vol. 152, 1999, pp. 550-583.

${ }^{26}$ Howell, L., Pember, R., Colella, P., Jessee, J., and Fiveland, W. A., "A Conservative Adaptive-Mesh Algorithm for Unsteady, Combined-Mode Heat Transfer Using the Discrete Ordinates Method," Numerical Heat Transfer, Vol. 35, 1999, pp. $407-430$.

${ }^{27}$ Groth, C. P. T., Zeeuw, D. L. D., Powell, K. G., Gombosi, T. I., and Stout, Q. F., "A Parallel Solution-Adaptive Scheme for Ideal Magnetohydrodynamics," Paper 99-3273, AIAA, June 1999.

${ }^{28}$ Groth, C. P. T., De Zeeuw, D. L., Gombosi, T. I., and Powell, K. G., "Global Three-Dimensional MHD Simulation of a Space Weather Event: CME Formation, Interplanetary Propagation, and and Interaction with the Magnetosphere," Journal of Geophysical Research, Vol. 105, No. A11, 2000, pp. 25,053-25,078.

${ }^{29}$ Sachdev, J., Groth, C., and Gottlieb, J., "A Parallel Solution-Adaptive Scheme for Predicting Multi-Phase Core Flows in Solid Propellant Rocket Motors," International Journal of Computational Fluid Dynamics, Vol. 19, No. 2, 2005 , pp. $159-177$.

${ }^{30}$ Douglas, C. C., Ern, A., and Smooke, M. D., "Numerical Simulation of Flames Using Multigrid Methods," Iterative Methods in Scientific Computation, edited by J. Wang, M. B. Allen, B. M. Chen, and T. Mathew, Vol. 4 of IMACS Series in Computational and Applied Mathematics, New Brunswick, 1998, pp. 149-154.

${ }^{31}$ Reynolds, W. and Fatica, M., "Stanford Center for Integrated Turbulence Simulations," Technical Paper 54-63, Stanford University, Marh/April 2000. 
${ }^{32}$ Medic, G., Kalitzin, G., You, D., Herrmann, M., Ham, F., van der Weide, E., Pitsch, H., and Alonso, J., "Integrated RANS/LES Computations of Turbulent Flow Through a Turbofan Jet Engine," Annual Research Briefs 275-285, Stanford University, 2006.

${ }^{33}$ Bell, J., Day, M., Almgren, A., Lijewski, M. J., and Rendleman, C. A., "Adaptive Numerical Simulation of Turbulent Premixed Combustion," Proceedings of the First MIT Conference on Computational Fluid and Solid Mechanics, June 2001.

${ }^{34}$ Bell, J., Day, M., Almgren, A., Lijewski, M. J., and Rendleman, C. A., "A Parallel Adaptive Projection Method for Low Mach Number Flows," International Journal for Numerical Methods in Fluids, Vol. 40, 2002, pp. 209-216.

${ }^{35}$ Bell, J., "AMR for Low Mach Number Reacting Flow," http://repositories.cdlib.org/lbnl/ LBNL-54351, 2004.

${ }^{36}$ Northrup, S. A. and Groth, C. P. T., "Solution of Laminar Diffusion Flames Using a Parallel Adaptive Mesh Refinement Algorithm," Paper 2005-0547, AIAA, January 2005.

${ }^{37}$ Gao, X. and Groth, C. P. T., "Parallel Adaptive Mesh Refinement Scheme for Turbulent Non-Premixed Combusting Flow Prediction," Paper 2006-1448, AIAA, January 2006.

${ }^{38}$ Gao, X. and Groth, C. P. T., "A Parallel Adaptive Mesh Refinement Algorithm for Predicting Turbulent Non-Premixed Combusting Flows," International Journal of Computational Fluid Dynamics, Vol. 20, No. 5, 2006, pp. 349-357.

${ }^{39}$ Wilcox, D. C., Turbulence Modeling for CFD, DCW Industries, La Cañada, 2002.

${ }^{40}$ Gordon, S. and McBride, B. J., "Computer Program for Calculation of Complex Chemical Equilibrium Compositions and Applications I. Analysis," Reference Publication 1311, NASA, 1994.

${ }^{41}$ McBride, B. J. and Gordon, S., "Computer Program for Calculation of Complex Chemical Equilibrium Compositions and Applications II. Users Manual and Program Description," Reference Publication 1311, NASA, 1996.

${ }^{42}$ Wilke, C. R., "A Viscosity Equation for Gas Mixtures," Journal of Chemical Physics, Vol. 18, 1950, pp. 517-519.

${ }^{43}$ W. C. Gardiner, J., Combustion Chemistry, Springer-Verlag New York Inc., Boca Raton, 1984.

${ }^{44}$ Westbrook, C. K. and Dryer, F. L., "Simplified Reaction Mechanisms for the Oxidation of Hydrocarbon Fuels in Flames," Combustion Science and Technology, Vol. 27, 1981, pp. 31-43.

${ }^{45}$ Barth, T. J., "Recent Developments in High Order K-Exact Reconstruction on Unstructured Meshes," Paper 93-0668, AIAA, January 1993.

${ }^{46}$ Roe, P. L., "Approximate Riemann Solvers, Parameter Vectors, and Difference Schemes," Journal of Computational Physics, Vol. 43, 1981, pp. 357-372.

${ }^{47}$ Einfeldt, B., "On Godunov-Type Methods for Gas Dynamics," SIAM Journal on Numerical Analysis, Vol. 25, 1988, pp. 294-318.

${ }^{48}$ Linde, T., "A Practical, General-Purpose, Two-State HLL Riemann Solver for Hyperbolic Conservation Laws," International Journal for Numerical Methods in Fluids, Vol. 40, 2002, pp. 391-402.

${ }^{49}$ Mathur, S. R. and Murthy, J. Y., "A Pressure-Based Method for Unstructured Meshes," Numerical Heat Transfer, Vol. 31, 1997, pp. 191-215.

${ }^{50}$ van Leer, B., Tai, C. H., and Powell, K. G., "Design of Optimally-Smoothing Multi-Stage Schemes for the Euler Equations," Paper 89-1933-CP, AIAA, June 1989.

${ }^{51}$ Sachdev, J. and Groth, C., "A Mesh Adjustment Scheme for Embedded Boundaries," Communications in Computational Physics, 2007, accepted in April 2007.

${ }^{52}$ McDonald, J. G. and Groth, C. P. T., "Numerical modeling of micron-scale flows using the Gaussian moment closure," Paper 2005-5035, AIAA, June 2005.

${ }^{53}$ Ivan, L. and Groth, C. P. T., "High-Order Central ENO Finite-Volume Scheme with Adaptive Mesh Refinement," Paper 2007-4323, AIAA, June 2007.

${ }^{54}$ Gropp, W., Lusk, E., and Skjellum, A., Using MPI, MIT Press, Cambridge, Massachussets, 1999.

${ }^{55}$ Aftomis, M. J., Berger, M. J., and Murman, S. M., "Application of Space-Filling Curves to Cartesian Methods for CFD," Paper 2000-1232, AIAA, January 2004.

${ }^{56}$ J.Laufer, "Investigation of Turbulent Flow in A Two-Dimensional Channel," Report 1053, NACA, 1951.

${ }^{57}$ J.Laufer, "The Structure of Turbulence in Fully Developed Pipe Flow," Report 1174, NACA, 1954.

${ }^{58} \mathrm{TNF}$, "International Workshop on Measurement and Computation of Turbulent Nonpremixed Flames," http://www.ca.sandia.gov/TNF/.

${ }^{59}$ Masri, A. R., Dibble, R. W., and Barlow, R. S., "The Structure of Turbulent Nonpremixed Flames of Methanol over a Range of Mixing Rates," Combustion and Flame, Vol. 89, 1992, pp. 167-185.

${ }^{60}$ Masri, A. R., Dibble, R. W., and Barlow, R. S., "Raman-Rayleigh Measurements in Bluff Body Stabilised Flames of Hydrocarbon Fuels," Twenty-fourth Symposium (International) on Combustion, The Combustion Institute, 1992, pp. 317-324. ${ }^{61}$ Masri, A. R., Dally, B. B., and Barlow, R. S., "The Structure of the Recirculation Zone of a Bluff-Body Combustor," Twenty-fifth Symposium (International) on Combustion, The Combustion Institute, 1994, pp. 1301-1308.

${ }^{62}$ Masri, A. R., Kelman, J. B., and Dally, B. B., "The Instantaneous Spatial Structure of the Recirculation Zone in Bluff-Body Stabilised Flames," Proceedings of the Combustion Institute, The Combustion Institute, 1998, pp. 1031-1038.

${ }^{63}$ Dally, B. B., Fletcher, D. F., and Masri, A. R., "Modelling of Turbulent Flames Stabilised on a Bluff-Body," Combustion Theory and Modelling, Vol. 2, 1998, pp. 193-219.

${ }^{64}$ Dally, B. B., Masri, A. R., Barlow, R. S., and Fiechtner, G. J., "Instantaneous and Mean Compositional Structure of Bluff-Body Stabilised Nonpremixed Flames," Combustion Theory and Modelling, Vol. 114, 1998, pp. 119-148.

${ }^{65}$ Turpin, G., , and Troyes, J., "Validation of a Two-Equation Turbulence Model for Axisymmetric Reacting and NonReacting Flows," Paper 2000-3463, AIAA, July 2000.

${ }^{66} \mathrm{Xu}$, J. and Pope, S. B., "PDF Calculations of Turbulent Nonpremixed Flames with Local Extinction," Combustion and Flame, Vol. 123, 200, pp. 281-307.

${ }^{67}$ Merci, B., Dick, E., Vierendeels, J., Roekaerts, D., and Peeters, T. W. J., "Application of a New Cubic Turbulence Model to Piloted and Bluff-Body Diffusion Flames," Combustion and Flame, Vol. 126, No. 1-2, 2001, pp. 1533-1556. 
${ }^{68}$ Liu, K., Pope, S., and Caughey, D., "Calculations of Bluff-Body Stabilized Flames Using a Joint Probability Density Function Model with Detailed Chemistry," Combustion and Flame, Vol. 141, 2005, pp. 89-117.

${ }^{69}$ Merci, B., An Unstructured Parallel Algorithm for Large Eddy and Conjugate Heat Transfer Simulations, Ph.D. thesis, Ghent University, 2000.

${ }^{70}$ Groth, C. P. T. and Northrup, S. A., "Parallel Implicit Adaptive Mesh Refinement Scheme for Body-Fitted Multi-Block Mesh," Paper 2005-5333, AIAA, June 2005.

${ }^{71}$ Northrup, S. A. and Groth, C. P. T., "Parallel Implicit Adaptive Mesh Refinement Algorithm for Solution of Laminar Combusting Flows," 14th Annual Conference of the CFD Society of Canada, Kingston, Ontario, Canada, July 16-18, 2006, July 2007.

${ }^{72}$ Northrup, S. A. and Groth, C. P. T., "Solution of Laminar Combusting Flows Using a Parallel Implicit Adaptive Mesh Refinement Algorithm," Fourth International Conference on Computational Fluid Dynamics, Ghent, Belgium, July 10-14, 2006, July 2006. 